\title{
Anatomia ultrassonográfica dos linfonodos abdominais de furões europeus hígidos ${ }^{1}$
}

\author{
Daniela Aparecida Ayres Garcia ${ }^{2 *}$, Luana Célia Stunitz da Silva ${ }^{3}$, Rogério Ribas \\ Lange $^{4}$ e Tilde Rodrigues Froes ${ }^{4}$
}

\begin{abstract}
Garcia D.A.A., Silva L.C.S., Lange R.R. \& Froes T.R. 2011.[Ultrasonographic anatomy of abdominal lymph nodes in the normal ferret.] Anatomia ultrassonográfica dos linfonodos abdominais de furões europeus hígidos. Pesquisa Veterinária Brasileira 31(12):1129-1132. Departamento de Medicina Veterinária, Universidade Federal do Paraná, Rua dos Funcionários 1540, Curitiba, PR 80035-050, Brazil. E-mail: daniapag@yahoo.com.br

In recent years the ferret (Mustela putorius furo) has become popular pet featuring a ever growing population in Brazil, and being increasingly found in veterinary clinics. This study aimed to evaluate the ultrasound anatomy of abdominal lymph nodes of healthy ferrets. We used 20 animals, nine of which males and eleven females, with overall mean age of three years. We located the mesenteric lymph nodes in $100 \%$ of the ferrets, the pancreatic-duodenal lymph nodes and the splenic ones in $55 \%$, the gastric lymph node in $20 \%$, and the hepatic lymph node in $5 \%$. We measured their lengths. It is concluded that the location and sonographic characteristics of the abdominal lymph nodes in ferrets are very similar to the abdominal lymph nodes of cats. This is a preliminary orientation for the location of abdominal lymph nodes of healthy ferrets.
\end{abstract}

INDEX TERMS: Ultrasound, ferret, lymph node.

RESUMO.- Nos últimos anos o furão (Mustela putorius furo) tornou-se um conhecido animal de estimação sendo observada uma população em constante crescimento no Brasil, e por conseqüência cada vez mais presente em clínicas veterinárias. 0 presente trabalho teve como objetivo avaliar a anatomia ultrassonográfica dos linfonodos abdominais de furões-europeus hígidos. Foram utilizados 20 animais, dentre os quais nove eram machos e onze fêmeas, com idade média total de três anos. Localizaram-se em $100 \%$ dos furões os linfonodos mesentéricos, em $55 \%$ dos animais os linfonodos pancreático-duodenal e esplênico, em $20 \%$ o linfonodo gástrico e em 5\% o linfonodo hepático. Conclui-se que a localização e características ultrassonográfica dos linfonodos abdominais em furões são muito similares aos linfonodos abdominais de gatos, sendo este estudo uma

\footnotetext{
${ }^{1}$ Recebido em 11 de julho de 2011.

Aceito para publicação em 2 de setembro de 2011.

${ }^{2}$ Mestranda do Programa de Pós-Graduação em Ciências Veterinárias, Universidade Federal do Paraná (UFPR), Rua dos Funcionários 1540, Bairro Juverê, Curitiba, PR 80035-050, Brasil. *Autor para correspondência: daniapag@yahoo.com.br

${ }^{3}$ Graduanda de Iniciação Científica, Departamento de Medicina Veterinária, UFPR, Curitiba, PR.

${ }^{4}$ Departamento de Medicina Veterinária, UFPR, Curitiba, PR.
}

orientação preliminar para a localização dos linfonodos abdominais de furões hígidos.

TERMOS DE INDEXAÇÃO: Ultrassom, furão-europeu, linfonodos.

\section{INTRODUÇÃO}

A crescente popularidade dos furões europeus (Mustela putorius furo) também conhecidos como ferretes que pertencem à ordem Carnivora e a família Mustelidae (Javorouski \& Passerino 2006) como animais de estimação e de modelos de pesquisa exige um maior conhecimento da sua anatomia ultrassonográfica. No entanto, ultrassonograficamente tem-se diversas informações sobre a glândula adrenal e muito pouco sobre os linfonodos desta espécie (Besso et al. 2000). Muitos ultrassonografistas têm dificuldade em diferenciar alguns linfonodos quando as adrenais estão aumentadas (Murphy et al. 1999).

A anatomia ultrassonográfica dos linfonodos em furões (Mustela putorius furo) é pouco conhecida e foi descrita apenas por Murphy et al. (1999) que relatou somente as características ultrassonográficas e macroscópicas do linfonodo mesentérico, esse localizado na junção cranial e caudal da veia mesentérica, envolvido pela gordura das raízes do mesentério, sendo que seu tamanho médio é de $12,4 \mathrm{~mm}$ (desvio padrão $\mathrm{sd}= \pm 2,4 \mathrm{~mm}$ ) de comprimento por $6,9 \mathrm{~mm}(\mathrm{sd}= \pm 2 \mathrm{~mm})$ de espessura. 
Em cães e gatos, informações sobre o tamanho, forma, localização e ecogenicidade dos linfonodos normais já foram amplamente descritas, estes tem a forma alongada e ecotextura homogênea e são ligeiramente hipoecóicos em comparação com a gordura mesentérica (Pugh 1994, Nyman et al. 2005, Schreurs et al. 2008).

Este trabalho tem como objetivo descrever a anatomia ultrassonográfica, localização e tamanho dos linfonodos abdominais de furões europeus hígidos.

\section{MATERIAL E MÉTODOS}

Vinte furões hígidos castrados, sendo nove machos e onze fêmeas, com idade média de três anos ( $\mathrm{sd}= \pm 1,21)$ sem sinais clínicos e hematológicos de alterações foram examinados pelo mesmo ultrassonografista com um aparelho dinâmico, bidimensional e com Doppler Modelo SONIX-OP, marca Ultrasonix (Viking Way, Richmond, BC, Canadá) e com transdutor linear de 14,0 MHz.

Os exames foram realizados após ampla tricotomia do abdome ventral, entre a região epigástrica e a região hipogástrica, compreendida entre o processo xifóide e os dois últimos pares de glândulas mamárias, estendendo-se bilateralmente à região dorsal. 0 animal era contido fisicamente pela pele ao redor da nuca e elevado verticalmente em relação à mesa, ficando com os membros livres ou contidos de forma suave pelo operador ou utilizando o antebraço como forma de apoio para o corpo do furão (Fig.1). Aplicou-se gel para contato do transdutor na pele do animal quando do momento do exame. Não foram empregados medicamentos anestésicos ou sedativos para o procedimento.

Durante o exame foi dada particular atenção a identificação dos linfonodos abdominais, com base em descrições anatômicas disponíveis (Fig.2). Os linfonodos hepáticos foram localizados na junção das veias esplênica e gastroduodenais com a veia porta

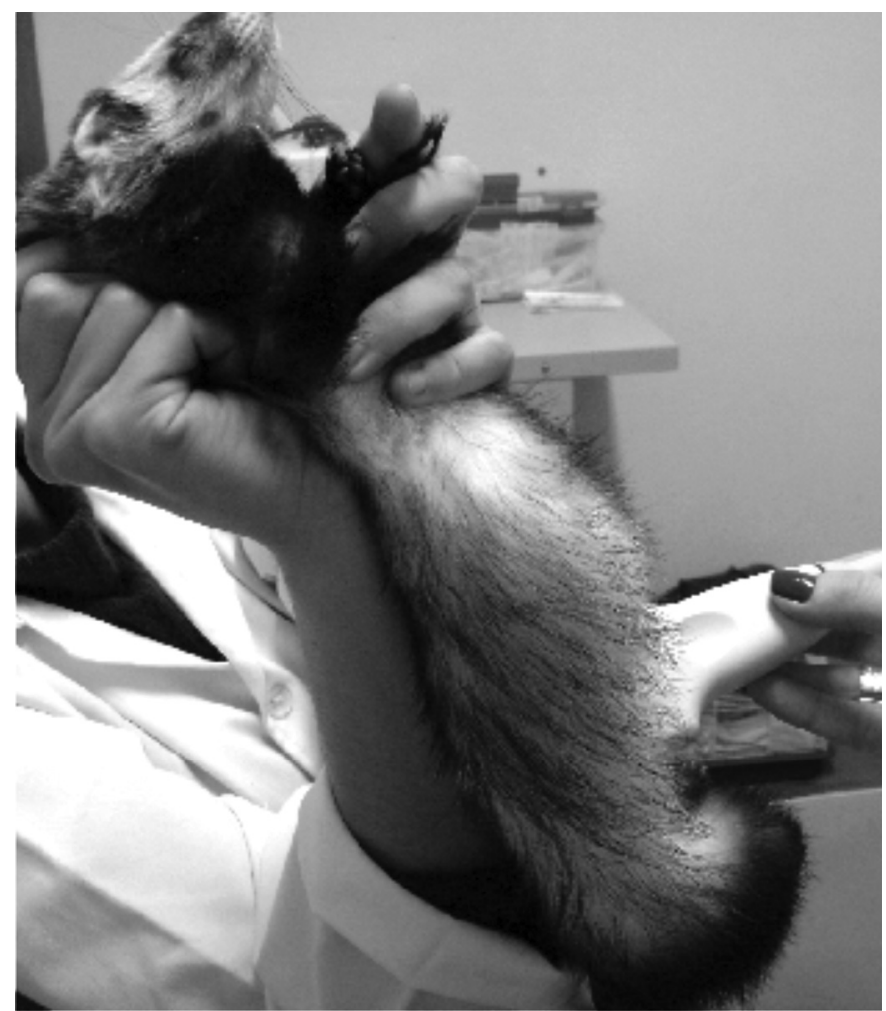

Fig.1. Aspecto do método de contenção do furão europeu para exame ultrassonográfico.

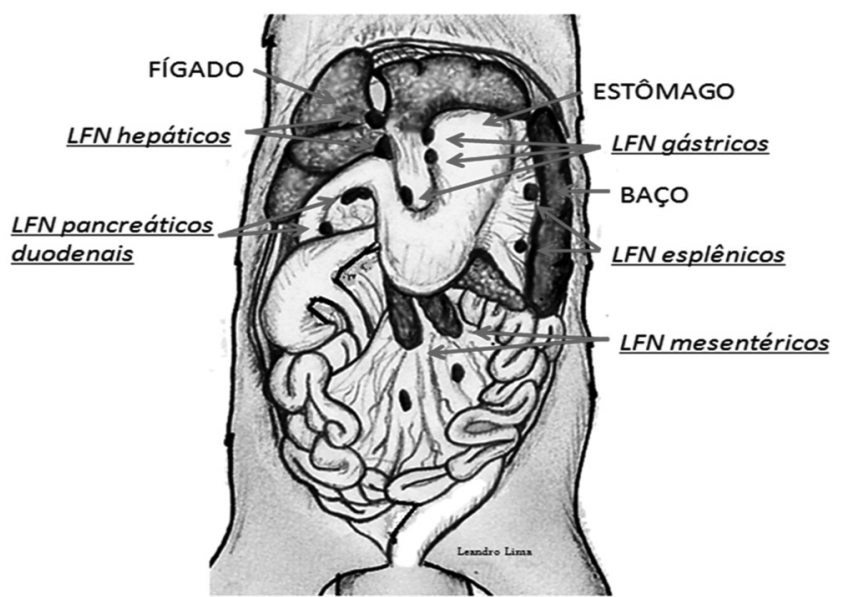

Fig.2. Esquema anatômico dos linfonodos abdominais encontrados ultrassonograficamente nos furões europeus.

e no hilo do fígado. Os linfonodos esplênicos estavam situados adjacentes aos vasos esplênicos ao redor do hilo esplênico. Os glânglios linfáticos gástricos foram visualizados incorporados no omento ao longo da curvatura menor do estômago, ao lado do cárdia ou ocasionalmente adjacentes ao piloro. Os linfonodos pancreáticos-duodenais se encontram caudal ao piloro, no encontro das veias pancreaticoduodenal cranial e gastroepiplóica direta. Pelo menos dois linfonodos mesentéricos estão localizados ao lado da artéria mesentérica cranial e na artéria mesentérica caudal foram identificados em região mesogástrica.

Os linfonodos foram reconhecidos pela ultrassonografia como sendo hipoecóicos, de estrutura alongada nas localizações anatômicas esperadas. Para cada linfonodo, quando identificado, foi medido seu comprimento. Posteriormente, a média dos comprimentos e espessuras máximos de cada linfonodo foram calculados pela média e seu desvio padrão, quando possível, no número de furões nos quais os mesmo foram identificados.

\section{RESULTADOS}

Os resultados estão apresentados no Quadro 1. Os linfonodos pancreático-duodenais, eventualmente identificados, localizavam-se na região de "zona pancreática" direita, utilizando-se a janela lateral direita; estes foram mensurados em 11 animais (Fig.3). No lado esquerdo, na região do hilo esplênico, foi possível identificar o linfonodo esplênico, observado também em 11 animais.

Em quatro animais foi visualizado o linfonodo gástrico, entre a flexura gástrica menor e o estômago, em apenas um animal todos os analisados fora possível a identificação ultrassonográfica do linfonodo hepático.

Quadro 1. Resultados obtidos por meio de exames ultrassonográficos dos linfonodos de furões europeus

(Mustela potorius furo) com freqüência, comprimento e desvio padrão

\begin{tabular}{ccc}
\hline Linfonodo & $\begin{array}{c}\text { Frequência de } \\
\text { observação }(\%)\end{array}$ & $\begin{array}{c}\text { Comprimento } \\
(\mathrm{mm})\end{array}$ \\
\hline Pancreáticos-duodenais & $55 \%$ & $5,29(\mathrm{sd}= \pm 1,32)$ \\
Esplênicos & $55 \%$ & $5,93(\mathrm{sd}= \pm 1,59)$ \\
Gástricos & $20 \%$ & $7,7(\mathrm{sd}= \pm 2,6)$ \\
Hepáticos & $5 \%$ & - \\
Mesentéricos & $100 \%$ & $10,18(\mathrm{sd}= \pm 2,36)$
\end{tabular}






Fig.3. Imagem ultrassonográfica da forma dos linfonodos pancreáticos-duodenais do furão europeu.

Constatou-se na avaliação do linfonodo mesentérico que em todos os animais o contorno e a ecogenicidade estavam normais. Em 16 animais o tamanho desses linfonodos estava normal, sendo que em quatro furões havia visivelmente um aumento quando comparado aos outros animais e ao descrito na literatura, estando localizado na região mesogástrica.

Para a toda a rede de linfonodos do sistema digestório identificável, a imagem ultrassonográfica característica constou-se de estruturas circulares ou alongadas (porém arredondadas), hipoecóicas, algumas delas circundadas por um leve halo ecogênico, e eventualmente em alguns animais estes linfonodos apresentavam-se com características mais anecóicas.

\section{DISCUSSÃO}

O linfonodo mesentérico foi localizado em todos os furões com as dimensões aproximadas de $10,18 \mathrm{~mm}$ (sd = $\pm 2,36 \mathrm{~mm}$ ) por $5,3 \mathrm{~mm}(\mathrm{sd}= \pm 1,39 \mathrm{~mm}$ ) sendo muito semelhantes numericamente com o encontrado por Murphy et al. (1999) em estudo conduzido para a realização de citologia aspirativa deste linfonodo em 28 furões normais obtendo-se medidas de $12,6 \mathrm{~mm}$ ( $\mathrm{sd}= \pm 2,6 \mathrm{~mm}$ ) por $7,6 \mathrm{~mm}$ ( $\mathrm{sd}$ $= \pm 2,0 \mathrm{~mm}$ ). A sua localização e relação topográfica foi semelhante à descrita pelos autores, por isso houve uma concordância com a colocação dos mesmos quando afirmaram que tal fato pode confundir o ultrassonografista quanto a uma suspeita de massa em adrenal esquerda, pois a pressão do transdutor pode causar uma alteração de sua posição levando a confusão diagnóstica (Murphy et al. 1999).

Notou-se que na mesma janela acústica utilizada inicialmente para a localização da região pancreática direita em cães e gatos pode-se visualizar o linfonodo pancreático-duodenal, e que segundo Schreurs et al. (2008) está localizado no aspecto caudal do piloro, local em que as veias pancreático-duodenal e gastroepiplóica direita se encontram, nos felinos, e visto que estes são similares morfologicamente aos furões, pode-se sugerir tanto pela literatura quanto pelas imagens ultrassonográficas vistas no presente estudo que tais linfonodos se encontram muito próximos ao ponto de referência anatômica descrita para os gatos, ou até mesmo no próprio ponto mencionado.

Os valores médios obtidos para o linfonodo pancreático-duodenal em furões foi de $5,29 \mathrm{~mm}(\mathrm{sd}= \pm 1,32 \mathrm{~mm}$ ) estes menores que os obtidos em gatos (Schereus et al. 2008). Deve-se tomar cuidado para não confundir estes linfondos com cistos ou mesmo nódulos pancreáticos, devido a sua localização anatômica, e aparência mais anecóica, até porque essa espécie também apresenta aparente maior incidência de tumores como insulinomas pancreáticos, sendo que as imagens previamente descritas como normais, poderiam ser confundidas.

Pode-se visualizar também o linfonodo gástrico, devido à sua localização e suas dimensões, que foi análogo aos achados de Etue et al. (2001), em gatos, ao citarem que o linfonodo gástrico está localizado craniomedialmente ao piloro, possuindo grandes dimensões. No trabalho de Schreurs et al. (2008) o valor de 5,1mm $(4,6-6,4 \mathrm{~mm})$ por $1,9 \mathrm{~mm}(1,9-1,9 \mathrm{~mm})$ é fornecido para o linfonodo gástrico, o que são similares aos valores de $7,7 \mathrm{~mm}(\mathrm{sd}= \pm 2,6 \mathrm{~mm})$ encontrados nos animais desse trabalho, apesar de não serem da mesma espécie. 0 achado do linfonodo hepático foi inconsistente em furões-europeus como em gatos, provavelmente devido a sua posição anatômica, devido a isso houve eventual dificuldade de se produzir imagem segura para perfeito registro de mensuração.

\section{CONCLUSÃO}

Conclui-se que a localização e características ultrassonográficas dos linfonodos abdominais em furões-europeus são muito similares aos linfonodos abdominais de gatos, podendo essa pesquisa ser considerada como base de comparação para futuros estudos.

\section{REFERÊNCIAS}

Besso J.G., Tidwell A.S. \& Gliatto J.M. 2000. Retrospective review of the ultrasonographic features adrenal lesions in 21 ferrets. Vet. Radiol. Ultrasound 41(4):345-352.

Etue S.M., Penninck D.G., Labato M.A., Pearson S. \& Tidwell A. 2001. Ultrasonography of the normal feline pancreas and associated anatomic landmarks: A prospective study of 20 cats. Vet. Radiol. Ultrasound 42(4):330-336.

Javorouski M.L. \& Passerino A. 2006. Carnivora: Mustelidae (ariranha, lontra, furão, irara, ferret), p.547-570. In: Cubas Z.L., Silva, J.C.R. \& Catão-Dias, J.L. (Eds), Tratado de Animais Selvagens. Roca, São Paulo. 
Murphy J.P., O’Brien R.T., Spaeth A., Sullivan L. \& Dubielzig R.R. 1999 Ultrasonography and fine needle aspirate cytology of the mesenteric lymph node in normal domestic ferrets (Mustela putorius furo). Vet. Radiol. Ultrasound 40(3):308-310.

Nyman H.T., Kristensen A.T., Skovgaard I.M. \& McEvoy F.J. 2005. Characterization of normal and abnormal canine superficial lymph nodes using gray-scale B-mode, color flow mapping, power, and spectral Doppler ultrasonography: A multivariate study. Vet. Radiol. Ultrasound 46:404410.

Pugh C.R. 1994 Ultrasonography examination of abdominal lymph nodes in the dog. Vet. Radiol. Ultrasound 35(2):110-115.

Schreurs E., Kathelijn V., Barberet V., Daminet S., Rudorf H. \& Saunders J.H. 2008. Ultrasonography anatomy of abdominal lymph nodes in the normal cat. Vet. Radiol. Ultrasound 49(1):68-72. 